\title{
A Multi-Gaussian Fuzzy Membership Function to the Algorithm Fuzzy GrowCut Applied to Segment Lesions in Mammography Images
}

\author{
Filipe R. Cordeiro, Beatriz Albuquerque, Valmir Macario
}

${ }^{1}$ Departamento de Computação (DC)

Universidade Federal Rural de Pernambuco (UFRPE)

filipe.rolimeufrpe.br, beatrizacbs@gmail.com, valmir.macario@ufrpe.br

\begin{abstract}
Segmentation of masses in mammography images is an important task to aid the accurate diagnosis of breast cancer. Although the quality of segmentation is crucial to avoid misdiagnosis, the segmentation process is a challenging task even for specialists, due to the presence of ill-defined edges and low contrast images. One of the techniques of state of the art for tumor segmentation is the Fuzzy GrowCut algorithm. In this work a study is performed on the behavior of this algorithm when using different membership functions for segmentation. Moreover, this research proposes a new membership function, called Multi-Gaussian, which improves the results of Fuzzy GrowCut with respect to those obtained through the use of classical functions.
\end{abstract}

\section{Introduction}

Breast cancer has been a growing problem to women around the world. According to World Health Organization (WHO), it is the most common type of cancer among women, with increasing mortality, becoming one of most lethal types of cancer [WHO 2015]. In $2015,570.000$ women died from breast cancer, corresponding to $15 \%$ of all deaths by cancer, between women, around the world [WHO 2015]. In Brazil, it is the primary cause of death by cancer between women, being estimated 59,700 new cases of cancer in 20182019, with an incidence of about 56.33 cases to each 100 thousand women, according to INCA [INCA 2018]. Early detection has a crucial impact on the tumor treatment success, being harder to cure in advanced stages [WHO 2015]. Several preventive methods have been developed, and one of most used methods to detect lesions is the digital mammography [Henriksen et al. 2018]. However, the mammography interpretation can be a challenging task even to specialists, once the analysis is influenced by several factors, such as quality of the image, radiologist experience and type of lesion. It is estimated that $12 \%$ to $30 \%$ of cases of cancer are missed due to bad mammographic interpretation [Evans et al. 2013].

In General, regions of lesion do not have a well-defined contour [Dheeba et al. 2014]. Therefore, seed based techniques [Cordeiro et al. 2013, Zheng et al. 2013], which the user label initial pixels, present better quality in the final segmentation. However, these techniques require higher specialist knowledge. Unsupervised techniques, such as those based on Active Contours [Rahmati et al. 2012] and Multilevel Thresholding [Chakraborty et al. 2012], present good segmentation to lesions with well-defined contours, but show difficulties to find spiculated or ill-defined edges. Supervised segmentation methods, such as those based on seeds, which the user label initial regions inside 
and outside the lesion, have obtained good segmentation quality related to the shape of objects [Tsochatzidis et al. 2017, Cordeiro et al. 2017a].

Zen et al. [Zheng et al. 2013] use the supervised algorithm Random Walker to segment lesions, obtaining good results to the metrics of sensibility and specificity. However, the algorithm Random Walker has parameters hard to be tuned and requires precise labeling of seeds in the internal and external regions of the lesion to work correctly.

Cordeiro et al. [Cordeiro et al. 2012] apply the GrowCut [Vezhnevets and Konouchine 2005] algorithm to segment lesions in mammography images, obtaining good segmentation results compared to state of the art. One of the GrowCut advantages is that it does not have parameters, but it still depends on the specialist labeling. Later, Cordeiro et al. Cordeiro et al. [Cordeiro et al. 2017b] modify the GrowCut algorithm, using Fuzzy Set Theory to reduce specialist labelling effort in the initialization of the algorithm, obtaining good results when compared to state of the art. The addition of membership function in the initialization of the algorithms showed good results. However, that analysis was performed using only Fuzzy-Gaussian membership function. This research aims to deepen the study of membership functions to verify what type of function fits better to perform segmentation of lesions in mammography images. To the best of our knowledge, this type of analysis was never performed in the literature.

\section{Membership Functions and Fuzzy GrowCut}

\subsection{Membership Functions}

In the classic set theory, the membership of an element to a set is related to the element belonging or not to this set. Unlike classic sets, the membership degree of a fuzzy set indicates the confidence of the element belonging to a set [Dubois et al. 2014], and this degree can vary from 0 to 1 [Rajasekaran and Pai 2011]. The 0 value means that an element does not belong to a specific set, and 1 means complete membership to the set. The values between 0 and 1 represent partial degrees of membership. Fuzzy sets are an important way to describe information that the elements can be represented by several degrees of membership. That is useful to describe concepts of the real world, e.g., to describe frontier regions of lesions in medical images, where there is not a well-defined border. This modeling is performed using membership functions, mapping the elements of a set at and assigning membership levels. Among the membership often used are triangular, trapezoidal, Gaussian, and generalized bell-shaped [Klir and Yuan 1995].

The Triangular membership function has a triangular shape and is defined by Equation 1:

$$
\mu_{\mathrm{tri}}(x)=\max \left(\min \left(\frac{x-a}{b-a}, \frac{c-x}{c-b}\right), 0\right),
$$

where $a$ and $c$ represent the position of the base of the triangle, in axis $x$, and $b$ is the position, also in axis $x$, in the top of the triangle. The functions max and min represent the maximum and minimum between the values, respectively. In this functions, the parameters must follow this order: $a<b<c$.

The Trapezoidal membership function is defined by Equation 2: 


$$
\mu_{\text {trap }}(x)=\max \left(\min \left(\frac{x-a}{b-a}, 1, \frac{d-x}{d-c}\right), 0\right)
$$

where $a$ and $d$ represent the position of wider base of trapezoid in axis $x$ and $b$, and $d$ is the lower base in the same axis, respectively. In this function, the parameters must follow this order: $a<b \leq c<d$.

The Gaussian membership function is defined by Equation 3:

$$
\mu_{\text {gauss }}(x)=e^{-\frac{(x-c)^{2}}{2 *(\beta)^{2}}}
$$

where $c$ and $\beta$ are the center and width of the fuzzy set, respectively. The number $x$ in the center of Gaussian region has maximum membership value and neighborhood membership values decrease gradually.

The Generalized Bell-Shaped function is defined by Equation 4:

$$
\mu_{\mathrm{sg}}(x)=\frac{1}{1+\left|\frac{x-c}{a}\right|^{2 b}},
$$

where $c, a$ and $b$ are the widths, the center and smoothness of the bell-shaped function, respectively.

\subsection{Multi-Dimensional Membership Function}

Let $l$ be the number of unidimensional membership functions $\mu_{k}(x), k=1, \ldots, l$ defined by a vector $x$ that creates a m-dimensional space. We can define a multidimensional membership function $\mu_{x}$ through the intersection between membership functions in each dimension [Abe 2012]. The intersection operators can be applied using the minimum operation, as described in Equation 5.

$$
\mu(x)=\min _{k=1, \ldots, l} \mu_{k}(x)
$$

The intersection can also be obtained using the product between the membership functions, as described in Equation 6.

$$
\mu(x)=\prod_{k}^{l} m_{k}(x)
$$

\subsection{Fuzzy GrowCut}

The algorithm Fuzzy GrowCut [Cordeiro et al. 2017b] is a supervised segmentation technique that requires the user to labels initial seeds related to the localization of the lesion in the mammography image. This technique uses a Fuzzy Gaussian membership function to estimate the membership function of pixels in the image to perform the segmentation. In the Fuzzy GrowCut, the pixels are labeled as background if the membership of a pixel 
to the background is higher than the membership compared to the foreground. The membership function associated to foreground and background are defined by Equation 7 and Equation 8, respectively:

$$
\begin{gathered}
\mu_{\mathrm{Obj}}(i)=\exp \left(-\frac{\left(x_{i}-x_{m}\right)^{2}}{2 \alpha_{x} s_{x}^{2}}\right) \exp \left(-\frac{\left(y_{i}-y_{m}\right)^{2}}{2 \alpha_{y} s_{y}^{2}}\right), \\
\mu_{\mathrm{Bkg}}(i)=1-\mu_{\mathrm{Obj}}(i),
\end{gathered}
$$

where $\mu_{\mathrm{Bkg}}(i)$ is the membership function degree associated with the uncertainty of the ith pixel belonging the image background, while $\mu_{\mathrm{Obj}}(i)$ is the membership function degree associated with the uncertainty of the ith pixel belonging to the foreground. These functions are Gaussian membership functions whose variables $x_{i}$ and $y_{i}$ corresponds to the coordinates of the ith pixel of the image, whereas $x_{m}$ and $y_{m}$ are coordinates of the center of mass of the labeled seeds. The values of $s_{x}$ and $s_{y}$ are the standard deviation of the initial seeds, and $\alpha_{x}$ and $\alpha_{y}$ are the tuning weights of the Gaussian function.

Figure 1 shows each step of segmentation process used by Fuzzy GrowCut. Figure 1(a) shows the region of interest (ROI) of the mammography image, which is submitted to initial labeling by the specialist. In Fuzzy GrowCut, it is necessary to perform only the labeling of regions inside ROI, as shown in Figure 1(b). Figure 1(c) shows the Gaussian region estimated by the membership function. The size of the region can be set through the parameter $\alpha$ of Equation 8. After the seeds have been initialized, the segmentation process starts, producing the segmented image, as shown in Figure 1(d), in which the segmented region is shown in green.

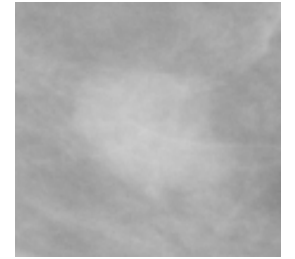

(a)

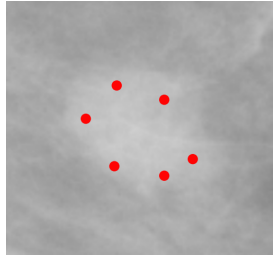

(b)

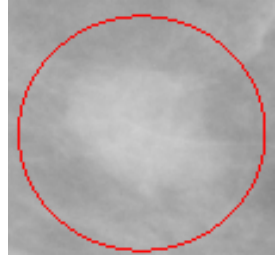

(c)

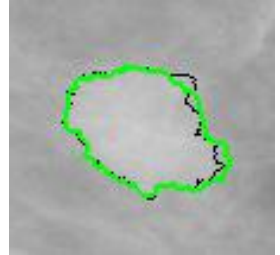

(d)

Figure 1. Stages of Fuzzy GrowCut. (a) ROI; (b) selection of seeds; (c) Fuzzy Gaussian membership function; (d) segmented image.

\section{Methodology}

\subsection{Project Flow}

The methodology developed in this research is illustrated through Figure 2.

The segmentation process starts with the mammography image from the database, followed by the selection of ROI, performed by the user. Next, the user labels the initial seeds, selecting the points where the lesion is located. These seeds serve as input to the Fuzzy GrowCut algorithm. In this research, the membership function to be used is also defined as the input to Fuzzy GrowCut. After this process, the segmented image is obtained and it is performed the analysis of the segmentation compared to the ground truth. Next, the database, the implemented membership functions, and metrics are described. 


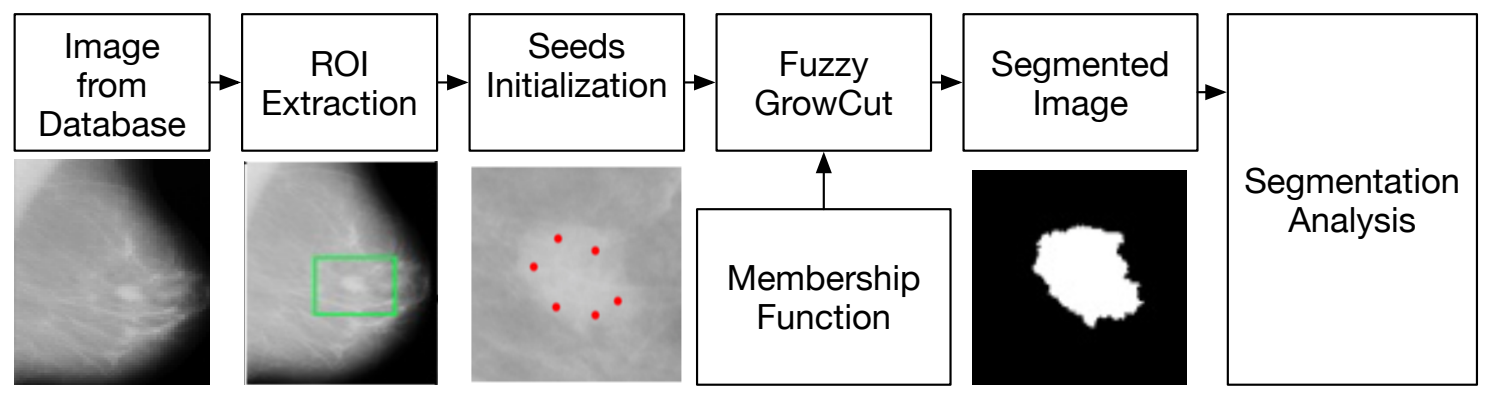

Figure 2. Project Flowchart

\subsection{Database}

In this research, it was used the Mini-MIAS [Suckling et al. 1994] public database to perform the analysis. The database consists of 322 mammography images with mediolateral oblique views, obtained from 161 patients. However, only 57 images of the database contain lesions classified as benign or malign. The other images of the database are classified as normal, having calcifications or asymmetry.

The ground truth, which corresponds to the specialist labeling, was constructed from the indications provided by the database using an auxiliary software and adaptive thresholding. From the selected images, the lesions have different classes: circumscribed, spiculated and indistinct. ROI selection and seeds labeling were done manually to perform the algorithm segmentation.

\subsection{Implemented Membership Function Models}

The membership function models implemented were the triangular, trapezoidal, Gaussian and generalized bell-shaped, described in section 2.2. To each one of them, it was used a composition through the operations of intersection of Equation 5 and Equation 6.

The Triangular membership function, defined by Equation 1, was implemented, tuning the parameters $a, b, c$ and $d$ according to the problem, and it is represented in two dimensions, according to Equation 9.

$$
\begin{gathered}
\mu_{\operatorname{tri}}(x, y)=\min \left(\mu_{\operatorname{tri}_{x}}(x), \mu_{\operatorname{tri}_{y}}(y)\right), \\
\mu_{\text {tri }_{k}}(u)=\max \left(\min \left(\frac{u-a_{k}}{\mathrm{CM}_{k}-a_{k}}, \frac{c_{k}-u}{c_{k}-\mathrm{CM}_{k}}\right), 0\right), \\
a_{k}=\mathrm{CM}_{k}-\sigma_{k} \cdot \beta, c_{k}=\mathrm{CM}_{k}+\sigma_{k} \cdot \beta,
\end{gathered}
$$

where $\mu_{\text {tri }}(x, y)$ defines the triangular membership function value in the position $(x, y)$ of the image. The values of $\mu_{\operatorname{tri}_{x}}$ and $\mu_{\operatorname{tri}_{y}}$ are the membership values to the axis $x$ and $y$, respectively. Therefore, the membership value is calculated through the minimum of the Triangular function applied in both dimensions. The $\mu_{\operatorname{tri}_{k}}(u)$ function defines the membership value to $k$ dimension, and it is expressed through the maximum and minimum operations. It is considered as the center of mass in axis $k$, expressed by $\mathrm{CM}_{k}$. To defined the bases of the triangle in the $k$ axis, represented by the parameters $a_{k}$ and $c_{k}$, it was used 
the center of mass and the standard deviation of the initial seeds, as shown in Equation 11. The variable $\beta$ sets how much the standard deviation in axis $k$, represented by $\sigma_{k}$, will influence the width of the triangle base in that dimension. To the intersection model used, the membership region is similar to a pyramid.

The trapezoidal membership function, defined by Equation 2, was represented in two dimensions and adapted to the problem, being calculated by Equation 12:

$$
\begin{gathered}
\mu_{\text {trap }}(x, y)=\min \left(\mu_{\operatorname{trap}_{x}}(x), \mu_{\operatorname{trap}_{y}}(y)\right), \\
\mu_{\operatorname{trap}_{k}}(u)=\max \left(\min \left(\frac{u-a_{k}}{b_{k}-a_{k}}, 1, \frac{d_{k}-u}{d_{k}-c_{k}}\right), 0\right), \\
a_{k}=\mathrm{CM}_{k}-\sigma_{k} \cdot \beta, \quad b_{k}=\mathrm{CM}_{k}-\sigma_{k} \cdot \frac{\beta}{2}, \\
c_{k}=\mathrm{CM}_{k}+\sigma_{k} \cdot \frac{\beta}{2}, \quad d_{k}=\mathrm{CM}_{k}+\sigma_{k} \cdot \beta,
\end{gathered}
$$

where $\mu_{\text {trap }}(x, y)$ is the trapezoidal membership function value to the position $(x, y)$ in the image, and $\mu_{\operatorname{trap}_{x}}$ and $\mu_{\operatorname{trap}_{y}}$ are the values corresponding to the axis $x$ and $y$, respectively. The membership value in axis $k$, represented as $\mu_{\operatorname{trap}_{k}}$, is defined by the maximum and minimum operations, using the variables $a_{k}, b_{k}, c_{k}$ and $d_{k}$, which define the base of the trapezium in the axis $k$. Those variables are defined by Equation 14 and were adapted to be automatically expressed through the center of mass and standard deviation in the axis $k$, represented by $\mathrm{CM}_{k}$ and $\sigma_{k}$, respectively. The variable $\beta$ is responsible for setting the influence of standard deviation in the definition of the base width of the trapezium in each axis.

The generalized bell-shape membership function, defined by Equation 4, was represented in two dimensions and adapted to the problem, being calculated by Equation 16.

$$
\begin{gathered}
\mu_{\operatorname{sinog}}(x, y)=\mu_{\operatorname{sinog}_{x}}(x) \cdot \mu_{\operatorname{sinog}_{y}}(y), \\
\mu_{\operatorname{sinog}_{k}}(u)=\frac{1}{1+\left|\frac{u-\mathrm{CM}_{k}}{2 \cdot \sigma_{k}}\right|^{2 b}},
\end{gathered}
$$

The generalized bell-shape function in two dimensions is calculated through the product of the function in each dimension, represented by $\mu_{\operatorname{sinog}_{x}}$ and $\mu_{\operatorname{sinog}_{x}}$, instead of being expressed by the minimum operation.

The Gaussian membership function, which is the model used in the Fuzzy GrowCut algorithm, was also implemented. However, the membership function was already showed in Equation 7. 
For this analysis, it was not used compositions with the union operations, because the resulting function was producing regions shapes without a defined border, what is bad to find a lesion in the image. The intersection models by minimization and product were chosen based on the most used models found in the literature.

Figure 3 shows the graph of implemented membership functions. The first line shows each function, in 3 dimensions, whereas the second one shows the membership region in 2 dimensions. The shape of membership regions influences on the mapping of the lesion region from the seed provided by the user.
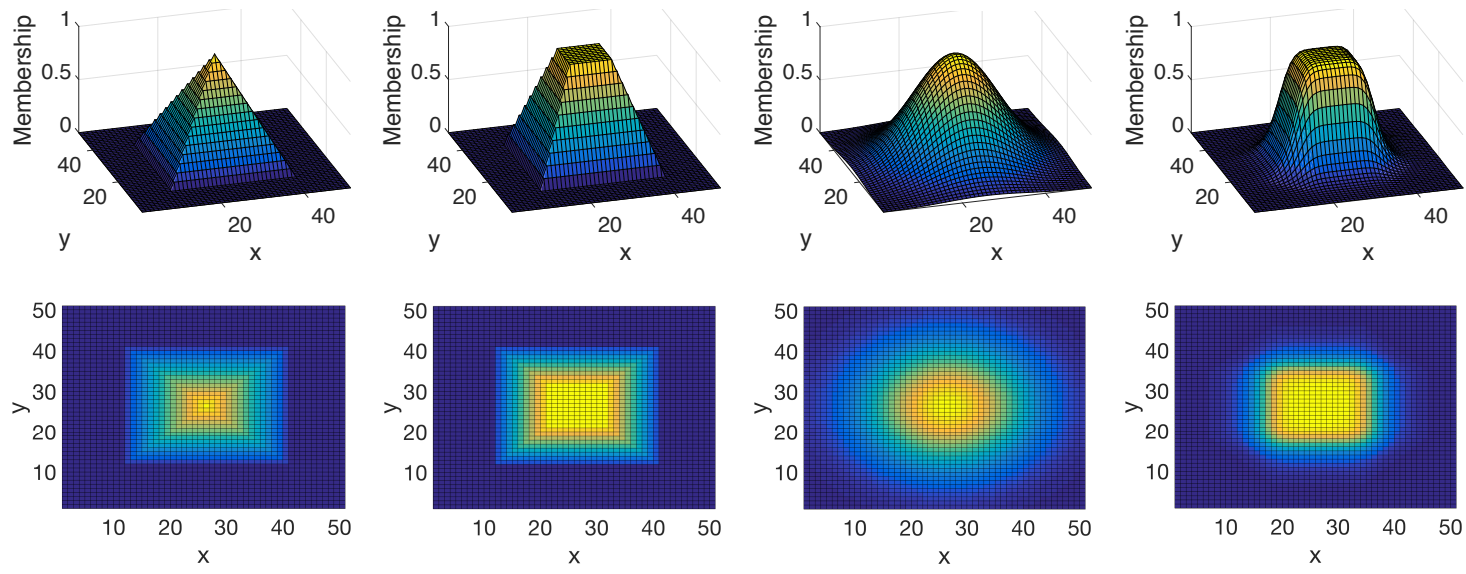

(a)

(b)

(c)

(d)

Figure 3. Implemented membership functions: (a) Triangular; (b) Trapezoidal; (c) Gaussian; (d) Generalized Bell-Shape.

\subsection{Proposed Model}

Besides the analysed membership functions, it was proposed a new model based on initial seeds location. The motivation behind the model creation is related to the shape of the lesions in mammography images and the shape of membership region using classical sets. Frequently, the lesions found does not have a shape similar to the ones presented in Figure 3, mainly for spicular lesions.

In the Fuzzy GrowCut algorithm, the center of mass of seeds provided by the user is used as the center of Gaussian region. In the proposed model, named as MultiGaussian model, it is generated a Gaussian function for each initial seed. Figure 4 shows the difference between the Gaussian model and the proposed Multi-Gaussian model.

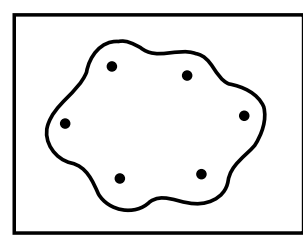

(a)

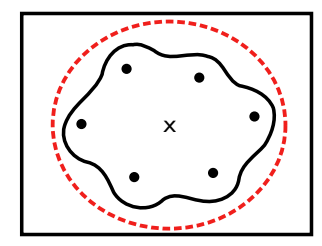

(b)

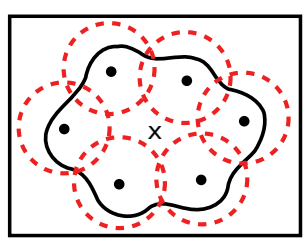

(c)

Figure 4. (a) lesion region with initial seeds; (b) region defined by Gaussian function; (c) region defined by Multi-Gaussian model.

Figure 4 (a) illustrates a tumor region with the initial seeds represented as black dots. Figure 4 (b) represents, in red, the region with a higher membership of pixel to the 
class of tumor. The $x$ symbol in the center of the image represents the center of mass of the provided seeds. Figure 4 (c) shows the proposed model, where each seed generates a Gaussian membership function. With the union of the generated Gaussian regions from each seed, it is possible to obtain a membership region closer to the edges of the legion, when compared to the Gaussian method.

The Multi-gaussian membership function to the position $(x, y)$ in the image, is represented as $\mu_{\mathrm{mg}}(x, y)=\max \left(\mu_{\text {seed }_{1}}(x, y)\right.$ and it is obtained by the operator of maximum between the memberships generated by each seed, expressed by $\operatorname{seed}_{k}$. The MultiGaussian membership function is calculated through Equation 18.

$$
\begin{gathered}
\mu_{\mathrm{mg}}(x, y)=\max \left(\mu_{\text {seed }_{1}}(x, y), \ldots, \mu_{\text {seed }_{k}}(x, y)\right), \\
\mu_{\text {seed }_{k}}(x, y)=e^{-\frac{\left(x-k_{x}\right)^{2}}{2 \cdot \alpha \cdot(\beta)^{2}}} \cdot e^{-\frac{\left(y-k_{y}\right)^{2}}{2 \cdot \cdot(\beta)^{2}}}
\end{gathered}
$$

The Equation 19 is an adaptation of Equation 7, that defines a Gaussian region for two dimensions. However, instead of using the center of mass of all seeds, it is used the position $(x, y)$ of each seed. Moreover, the width of Gauss region not anymore defined by the standard deviation of all seed, but by the constant $\beta$. The proposed model is adaptable to the number of seeds provided by the user. It is expected that the more seeds are provided, better is the representation of the model.

\subsection{Metrics}

To perform the evaluation of segmentation, some metrics of state of the art to analyze medical images were implemented: Sensibility, Specificity, Jaccard Index, Dice, and Balanced Accuracy. Those metrics are calculated from the rates of True Positive (TP), True Negative (TN), False Positive (FP), and False Negative (FN) found in the segmented images. Next, it is described each metric.

Sensibility (SE) [Taha and Hanbury 2015] represents the effectiveness of the algorithm to correctly classify the pixels from the object in the image. The sensibility can be described through Equation 20.

$$
\mathrm{SE}=\frac{\mathrm{TP}}{\mathrm{TP}+\mathrm{FN}}
$$

Specificity (SP) [Taha and Hanbury 2015] represents the effectiveness of the algorithm to correctly classify the pixels from the background in the image. The specificity can be described through the Equation 21.

$$
\mathrm{SP}=\frac{\mathrm{TN}}{\mathrm{FP}+\mathrm{TN}}
$$

Balanced Accuracy (BAC) [Hirschauer et al. 2015] is the average between specificity and sensibility. The more the value of BAC the more balanced is the relation between SE and SP. The balanced accuracy is calculated according to Equation 22 


$$
\mathrm{BAC}=\frac{\mathrm{SE}+\mathrm{SP}}{2}
$$

Jaccard index (J) [Taha and Hanbury 2017] represents the similarity index between a segmented image and its respective ground truth. The Jaccard index is calculated through Equation 23.

$$
\mathrm{J}=\frac{\mathrm{TP}}{\mathrm{TP}+\mathrm{FN}+\mathrm{FP}}
$$

The Dice metric [Lu et al. 2009] is another metric frequently used to evaluate segmentation algorithms, and it is defined by Equation 24:

$$
\text { Dice }=\frac{2 \cdot \mathrm{TP}}{\mathrm{S}+\mathrm{GT}}
$$

where $S$ is the area of the Segmented object, and $G T$ is the area of ground truth.

\section{Results}

An initial study was performed to find the best values of parameters to each membership function. The parameter settings were done using the metrics described in section 3.5. Table 1 shows the best values found for each membership function:

Table 1. Best parameters values to the membership functions.

\begin{tabular}{ll}
\hline Function & Parameters \\
\hline Gaussian & $\alpha=10$ \\
Triangular & $\beta=6$ \\
Trapezoidal & $\beta=4$ \\
Generalized Bell & $b=6$ \\
Proposed & $\beta=35$ \\
\hline
\end{tabular}

The segmentation results were analysed in respect to the 57 database images, where it was extracted the ROI and labeled the initial seeds. Next, segmentation results of Fuzzy GrowCut were analysed for each membership function, comparing to the ground truth of each image. Figure 5 shows the segmentation results to the images $m d b 12, m d b 28$ e $m d b 145$ of Mini-MIAS database.

The first column of Figure 5 shows the region of interest of the original image, whereas the columns (b)-(f) represent the segmentation results using Gaussian, Triangular, Trapezoidal, Generalized Bell-Shaped and proposed membership functions, respectively. In Figure 5, the black contour represents the specialist annotation, corresponding to the ground truth, while the green contour is the segmentation of each approach. It is interesting to notice that the results of the Triangular and the Trapezoidal functions are identical, because the best parameter of the Trapezoidal function made it approximate to the Triangular function, producing the same results. It is possible to observe that the Fuzzy GrowCut with Gaussian membership function, that is the original approach, has 

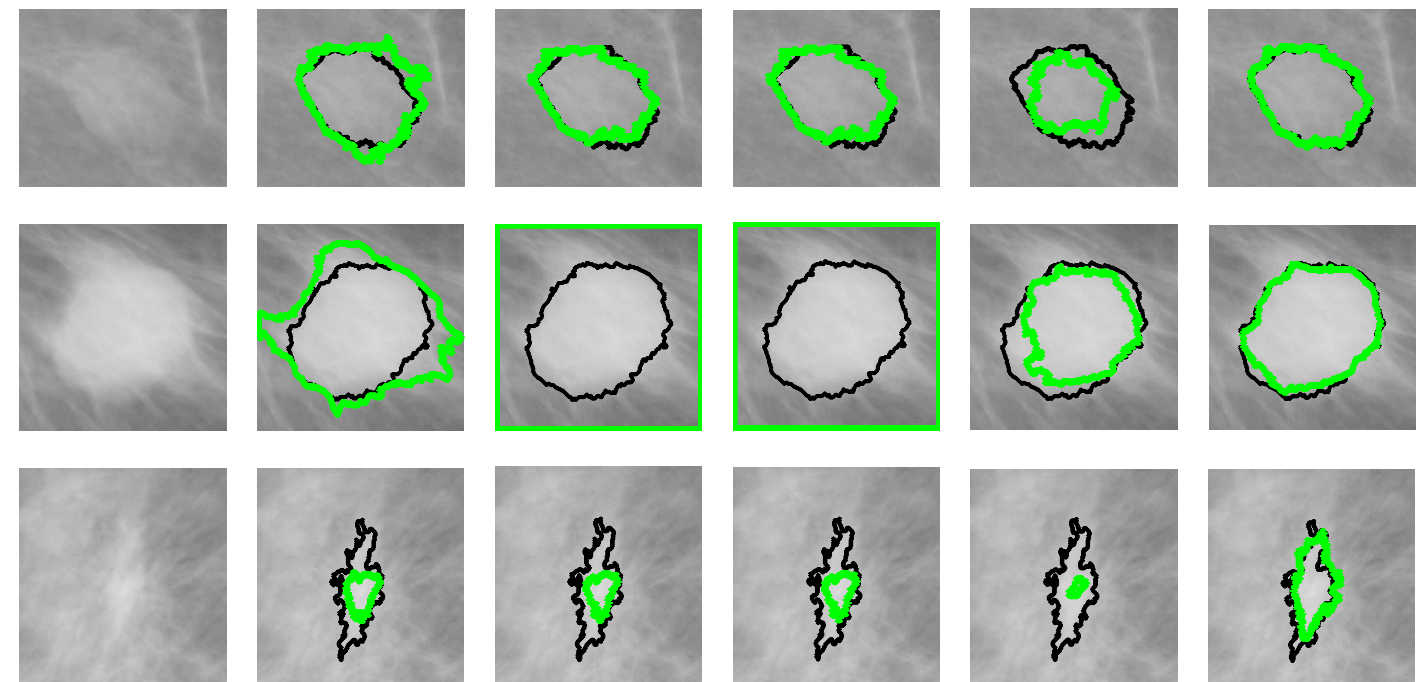

(a)

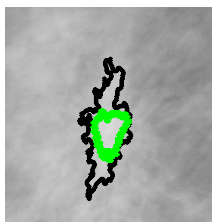

(b)

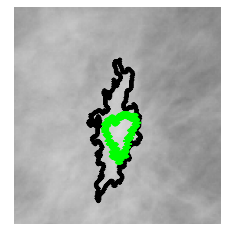

(c)

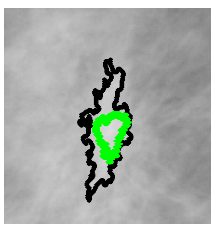

(d)

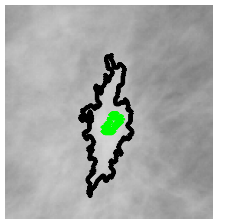

(e)

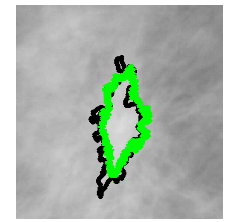

(f)

Figure 5. Segmentation results of GrowCut, using the membership functions analysed. (a) ROI; (b)-(f) Gaussian, Triangular, Trapezoidal, Generalized Bellshaped and proposed functions, respectively.

difficulties to segment some lesions. The same happens with the Generalized Bell-Shaped function. When the proposed Multi-Gaussian model is used, results closer to the ground truth to some images can be found, as shown in Figure 5. This difference happens mainly in lesions of type spiculated, as shown in the third line of Figure 5.

Table 2 shows the quantitative results of each analysed metric. The values in bold show the best values to each metric.

Table 2. Results of the metrics for each membership function.

\begin{tabular}{llllll}
\hline Function & SE & SP & BAC & J & Dice \\
\hline Gaussian & $\mathbf{0 . 7 4}$ & 0.88 & 0.81 & 0.62 & 0.75 \\
Triangular & 0.66 & 0.85 & 0.76 & 0.55 & 0.69 \\
Trapezoidal & 0.66 & 0.85 & 0.76 & 0.55 & 0.69 \\
Generalized Bell-Shaped & 0.35 & $\mathbf{1}$ & 0.67 & 0.34 & 0.48 \\
Proposed & 0.72 & 0.98 & $\mathbf{0 . 8 5}$ & $\mathbf{0 . 6 3}$ & $\mathbf{0 . 7 5}$ \\
\hline
\end{tabular}

From Table 2 we can see that the proposed method has better results for the metrics of balanced accuracy, Jaccard Index and Dice. Although the Gaussian method has a higher Sensibility, the Specificity is lower, resulting in lower BAC. Although the Generalized Bell-Shaped Function has high Specificity, it was not efficient to map the region of lesions correctly, resulting in low Sensibility. The proposed Multi-Gaussian method achieves better results by allowing to estimate representation of the lesion that is closer to the real contour. That happens due to the better use of information of seeds, allowing a better segmentation. The results obtained in this project can be extended to other seed-based segmentation methods and other medical images databases. 


\section{Conclusion}

This research performed an analysis of different membership functions applied to the GrowCut algorithm, that is an algorithm used to segment lesions in mammography images. The analysis was performed with 57 images of the Mini-MIAS database, using Gaussian, Triangular, Trapezoidal and Generalized Bell-Shaped membership functions. Moreover, it was proposed a new membership function, named Multi-Gaussian.

The analysis results showed that the proposed model allowed the Fuzzy GrowCut to obtain better segmentation results when compared to traditional membership functions. It was observed that the Multi-Gaussian model could map the region of the lesion better, obtaining better values to the metrics of BAC, Jaccard and Dice.

With this research, it was possible to observe that the use of membership function has a high impact in the Fuzzy GrowCut algorithm and that the proposed membership model can be applied to improve other seed-based techniques and extended to other databases.

\section{Acknowlegements}

The authors thank the support received by The Brazilian National Council for Scientific and Technological Development - CNPQ.

\section{References}

Abe, S. (2012). Pattern classification: neuro-fuzzy methods and their comparison. Springer Science \& Business Media.

Chakraborty, J., Mukhopadhyay, S., Singla, V., Khandelwal, N., and Rangayyan, R. M. (2012). Detection of masses in mammograms using region growing controlled by multilevel thresholding. In Computer-Based Medical Systems (CBMS), 2012 25th International Symposium on, pages 1-6, Roma. IEEE.

Cordeiro, F. R., Bezerra, K. F. P., and dos Santos, W. P. (2017a). Random walker with fuzzy initialization applied to segment masses in mammography images. In ComputerBased Medical Systems (CBMS), 2017 IEEE 30th International Symposium on, pages 156-161. IEEE.

Cordeiro, F. R., Lima, S. M., Silva-Filho, A. G., and Santos, W. (2012). Segmentation of mammography by applying extreme learning machine in tumor detection. In Intelligent Data Engineering and Automated Learning-IDEAL 2012, pages 92-100. Springer.

Cordeiro, F. R., Santos, W. P., and Silva-Filho, A. G. (2013). Segmentation of mammography by applying growcut for mass detection. women, 1:3.

Cordeiro, F. R., Santos, W. P. d., and Silva-Filho, A. G. (2017b). Analysis of supervised and semi-supervised growcut applied to segmentation of masses in mammography images. Computer Methods in Biomechanics and Biomedical Engineering: Imaging \& Visualization, 5(4):297-315.

Dheeba, J., Singh, N. A., and Selvi, S. T. (2014). Computer-aided detection of breast cancer on mammograms: A swarm intelligence optimized wavelet neural network approach. Journal of Biomedical Informatics, 49:45-52. 
Dubois, D. J., Prade, H., and Yager, R. R. (2014). Readings in fuzzy sets for intelligent systems. Morgan Kaufmann.

Evans, K. K., Birdwell, R. L., and Wolfe, J. M. (2013). If you don't find it often, you often don't find it: Why some cancers are missed in breast cancer screening. PloS one, $8(5): 364-366$.

Henriksen, E. L., Carlsen, J. F., Vejborg, I. M., Nielsen, M. B., and Lauridsen, C. A. (2018). The efficacy of using computer-aided detection (cad) for detection of breast cancer in mammography screening: a systematic review. Acta Radiologica, page 0284185118770917.

Hirschauer, T. J., Adeli, H., and Buford, J. A. (2015). Computer-aided diagnosis of parkinson's disease using enhanced probabilistic neural network. Journal of medical systems, 39(11):179.

INCA (2018). Estimativa 2018 - Incidência de Câncer no Brasil, volume 1.

Klir, G. and Yuan, B. (1995). Fuzzy sets and fuzzy logic, volume 4. Prentice hall New Jersey.

Lu, Y., Radau, P., Connelly, K., Dick, A., and Wright, G. A. (2009). Segmentation of left ventricle in cardiac cine mri: An automatic image-driven method. In International Conference on Functional Imaging and Modeling of the Heart, pages 339-347. Springer.

Rahmati, P., Adler, A., and Hamarneh, G. (2012). Mammography segmentation with maximum likelihood active contours. Medical image analysis, 16(6):1167-1186.

Rajasekaran, S. and Pai, G. V. (2011). Neural Networks, Fuzzy Logic and Genetic Algorithms. PHI Learning Private Limited.

Suckling, J., Parker, J., Dance, D., Astley, S., Hutt, I., Boggis, C., Ricketts, I., Stamatakis, E., Cerneaz, N., Kok, S.-L., et al. (1994). The mammographic image analysis society digital mammogram database. 2nd International Workshop on Digital Mammography.

Taha, A. A. and Hanbury, A. (2015). Metrics for evaluating 3d medical image segmentation: analysis, selection, and tool. BMC medical imaging, 15(1):29.

Taha, A. A. and Hanbury, A. (2017). Evaluation metrics for medical organ segmentation and lesion detection. In Cloud-Based Benchmarking of Medical Image Analysis, pages 87-105. Springer.

Tsochatzidis, L., Zagoris, K., Arikidis, N., Karahaliou, A., Costaridou, L., and Pratikakis, I. (2017). Computer-aided diagnosis of mammographic masses based on a supervised content-based image retrieval approach. Pattern Recognition, 71:106-117.

Vezhnevets, V. and Konouchine, V. (2005). GrowCut - Interactive Multi-Label N-D Image Segmentation By Cellular Automata. Graphicon, pages 150-156.

WHO (2015). WHO position paper on mammography screening. World Health Organization.

Zheng, S.-W., Liu, J., and Liu, C.-C. (2013). A random-walk based breast tumors segmentation algorithm for mammograms. Computer, Consumer and Control (IJ3C), International Journal on. 